Niniejsza publikacja jest dostęna na licencji Creative Commons. Uznanie autorstwa-Użycie niekomercyjne-Bez utworów zależnych 3.0 Polska. Pewne prawa zastrzeżone na rzecz autora. Zezwala się na wykorzystanie publikacji zgodnie z licencja - pod warunkiem zachowania niniejszej informacji licencyjnej oraz wskazania autora jako właściciela praw do tekstu. Treść licencji jest dostępna na stronie: http://creativecommons.org/licenses/by-nc-nd/3.0/pl/

Lingwistyka Stosowana 18: 3/2016, 151-164

\title{
Ewa ŻEBROWSKA
}

Universytet Warszawski

\section{Text und Bild im Transfer des medizinischen Fachwissens}

\begin{abstract}
:
Text and image in medical technical knowledge transfer

Knowledge, its production, storage and transfer are defining features of modern societies. The communication of information and knowledge is related, in the most important areas of life, with the progressive specialization, and thus it has also become a key task in the knowledge societies. The paper poses the question of how technical knowledge is communicated between experts and lay people in the field of medicine, which linguistic and non-linguistic means are applied in the process, and finally which visualization techniques contribute to improving the effectiveness and efficiency of professional communication.
\end{abstract}

Bei der Steigerung, Verwendung und Organisation des Wissens in der menschlichen Kulturgeschichte kommt den sprachlichen und textuellen Fähigkeiten von Menschen eine wichtige Rolle zu. Historisch und systematisch gesehen gelten Texte und Bücher als sprachliche Konstitutionsformen von Wissen sowie als fassbare Manifestationsformen des Wissens und des kulturellen Gedächtnisses, das sie in einer ganz konkreten Form zum Ausdruck bringen und zugänglich machen. Mit Hilfe von Texten versprachlichen die Menschen das Wissen, erzeugen „Wissenswelten“ und bringen eine kognitive Ordnung in die Welt, so G. Antos (2007: 41). Neue Technologien und neue Medien, dank denen Informations- und Kommunikationsmöglichkeiten wesentlich erweitert worden sind, bringen auch neue Entwicklung mit sich. „Informationen und Wissen sind heute zu einem Gemeingut aller (ausgebildeten) Menschen (mit Zugang zu IuK-Technologien) geworden." (G. Antos 2003: 481). Vor dem Hintergrund dieser Entwicklung wird das Wissen zunehmend als wertvolle Ressource betrachtet. Diese Meinung ist in den Wirtschaftswissenschaften verbreitet, in denen das Wissen sogar als vierter Produktionsfaktor verstanden wird. An Bedeutung gewinnen daher solche Ideen, wie Patentierung von zukunftsrelevantem Wissen, die juristische Aneignung, Verwertung und Vermarktung (G. Antos 2003: 481). Auch S. Grucza (2012: 105) betont die Tatsache, dass dem Wissen ein ökonomischer Wert zugeschrieben wird und dass das Wissen als Aktiva des intellektuellen Kapitals betrachtet wird, denn in modernen Wirtschaftsmodellen gewinnen nicht mehr die Industrie, Landwirtschaft und Dienstleistungen, sondern eben der Besitz von (Fach-)Wissen, ihr Erwerb, ihre Erzeugung, Generierung, Verarbeitung, Ver- 
waltung und Nutzung an Bedeutung (Informations- bzw. Wissenserzeugung und verteilung). Neue Informations- und Kommunikationstechnologien erleichtern diese Prozesse und haben sogar zur (technischen) Informationsrevolution beigetragen, so dass die moderne Wirtschaft als Informationswirtschaft und die moderne Gesellschaft als Informationsgesellschaft bezeichnet werden (S. Grucza 2012: 104). Man muss jedoch stets im Auge behalten, dass das Wissen in Produkten, in diversen Speicherträgern nicht enthalten ist, eingebaut oder gespeichert wird. Wissen ist immer das Wissen einer konkreten Person, ihre immanente Eigenschaft. Deshalb bedeuten solche Ausdrücke, wie Wissenszugänglichkeit, -management, -verarbeitung usw. Zugänglichkeit zum Wissen einer konkreten Person/konkreter Personen (S. Grucza 2012: 107).

Das Wissen wird nicht nur vom ökonomischen und informationstechnischen Standpunkt aus betrachtet, sondern es wird in der Psychologie, den Kognitionswissenschaften und in der Epistemologie zum Forschungsobjekt. Die (Fachsprachen-)Linguistik könnte hier die Rolle der Sprache bei der Erkenntnis selbst sowie bei der Vermittlung deren Ergebnisse erforschen. Ich stimme mit A. Ziems überein (S. Grucza 2012: 100), wenn er folgende Fragestellungen innerhalb der sprachwissenschaftlichen Analysepraxis formuliert: Wie schaffen wir mit sprachlichen Mitteln Wissen? Wie lässt sich das so geschaffene Wissen linguistisch analysieren?

Es wird heutzutage nicht nur danach gefragt, wie das Wissen konstituiert wird, sondern auch danach, unter welchen Bedingungen sich das Wissen erfolgsreich kommunizieren lässt. Nach dem Postulat von G. Antos (2003: 483) sollte eine interdisziplinäre Erforschung des Wissenstransfers unter dem Namen >Transferwissenschaft< etabliert werden, im Rahmen deren auch der Linguistik ihre Aufgaben zukommen, und zwar die Erforschung der Wissenskommunikation selbst.

Die Transferwissenschaft erforscht die kulturellen, sozialen, kognitiven, sprachlichmedialen und emotionalen Bedingungen, die medialen Wege sowie Prinzipien und Probleme der Wissensproduktion und -rezeption unter dem Gesichtspunkt ihrer strukturellen und sozialen Vernetzung, ihrer Relevanz für Nicht-Experten und den Chancen ihres globalen sowie gruppen- und zielspezifischen Transfers. (G. Antos 2003: 484)

G. Antos (2003: 480) zufolge galt bis heute, dass Wissenschaft, Wirtschaft und Gesellschaft die Wissensproduktion erforschen und optimieren, also die Frage nach ihrer Art und dem Umfang stellen. Für die Zukunft jedoch wird diese Aufgabe um eine zusätzliche ergänzt, und zwar auch der Zugang zum Wissen soll erforscht und optimiert werden. Es erscheint also die Frage, wie der Zugang zu dem (fach-)wissenschaftlichen, technischen, wirtschaftlichen und kulturellen Wissen (um genauer zu sagen: zu seinen Exponenten) beschaffen ist und wie er nachhaltig offen gehalten werden kann (G. Antos 2003: 481). In den Zeiten von Informationsflut und einer explosionsartigen Wissensproduktion wachsen beispielsweise technischorganisatorische Barrieren (Probleme des Retrievals im Internet), Datenmüll, ihre Unübersichtlichkeit, fehlendes Hintergrundwissen, fehlende hermeneutische Fähigkeiten sowie Allgemein- und Spezialbildung. Darüber hinaus ist eine wichtige Bedingung für einen potentiellen Transfer von Wissen das Metawissen (Zugriffswissen), die die Existenz, die Struktur, den Inhalt und die Implikationen eines Wissens- 
bestandes umfasst. Die Transferwissenschaft sollte sich schließlich auch damit befassen, im Einzelnen mit Bedingungen, Prinzipien, Formen, Strategien, Erfolgschancen, mit dem Ziel, eine nicht eingeschränkte Verfügbarkeit von (Fach-, Sondern-)Wissen für alle daran Interessierten zu sichern (G. Antos 2003: 484).

Das, was sich transferieren lässt, also das Wissen, ist eine spezifische immanente Eigenschaft eines jeden Menschen, d.h. seines Gehirns (auch anderer Lebewesen) und existiert nicht selbständig. Es entzieht sich jeder direkten Beobachtung, denn es lassen sich nur konkrete Handlungen und deren Ergebnisse beobachten, zu denen Fachtexte gehören. Den direkten Zugang zu diesem Wissen als einer immanenten Eigenschaft einer konkreten Person hat allein die jeweilige Person, die es besitzt. Die anderen können nur indirekten Zugang dazu haben, der durch die materiellen Exponenten, den Ersatz, die Repräsentation des Wissens möglich ist. Als Wissensexponenten gelten Texte, Tabellen, Endprodukte, Effekte der Anwendung des Wissens etc., also ausschließlich das, was eine materielle Form annehmen kann. Dies sind in erster Linie sprachliche Ausdrücke - Wörter, Sätze, Texte, aber darüber hinaus auch andere Mittel, wie Bilder, Graphiken, Diagramme usw., die sprachliche Elemente bebildern und visualisieren. Nur zu diesen Exponenten haben wir einen direkten Zugang, und nur sie können wir verwalten, verarbeiten, managen. Das Wissen selbst kann von den einzelnen konkreten Personen erzeugt und verarbeitet werden (S. Grucza 2012: 107f.).

Jedes Fachwissen ist immer ein Wissen über etwas Konkretes, ein konkretes Objektwissen, es gibt kein Wissen als solches. Die Kategorisierung des Fachwissens kann also danach vorgenommen werden, worauf es sich bezieht: auf welchen Gegenstand (Objekt), auf welche Eigenschaften des Gegenstandes, auf welche Relationen zwischen den Eigenschaften des Gegenstandes und schließlich auf welche Relationen zwischen den einzelnen Elementen des Gesamtbestandes. Gegenstände, auf die sich das Fachwissen bezieht, existieren jetzt, haben existiert oder werden existieren. Dementsprechend gibt es das diagnostische Fachwissen, das anagnostische Fachwissen und das prognostische Fachwissen. Nach einer der Unterscheidungen gibt es das vererbte (genetische) Wissen und das erworbene (kulturbezogene) Wissen (S. Grucza 2012: 158). Es seien auch hier genannt: das sprachliche und außersprachliche Wissen; das theoretische (deskriptive, explikative, prognostische usw.) und praktische, applikative; das bewusste und unbewusste Wissen; das Grundwissen (hier auch: das Fachwissen) und das Metawissen (S. Grucza 2012: 121). Viele Elemente des (Fach-)wissens der einzelnen Menschen wie auch der Gemeinschaften über die gegebene Wirklichkeit oder Elemente seines Verständnisses stammen aus der Kenntnis der diese Wirklichkeit betreffenden Fachsprachen, und nicht aus anderen Quellen, wie etwa Erfahrung, Deduktion oder Gedankengänge.

Sowohl die Erzeugung des Fachwissens als auch die Formulierung der Fachtexte, in denen die Fachleute ihr Fachwissen zum Ausdruck bringen, sei allein mit Hilfe der Fachsprachen möglich. S. Grucza (2012: 101f.) zufolge erfüllen die Fachsprachen nicht nur kommunikative, sondern auch kognitive Funktionen. Die Fachsprachen werden von ihren Anwendern, also von konkreten Fachleuten bewusst gleichzeitig mit dem Erkennen und/oder Entstehen eines bestimmten Bereichs der Wirk- 
lichkeit gebildet. Fast alle Denkprozesse werden in der Sprache durchgeführt und in ihr werden mentale Konstruktionen entwickelt. Auch K. Ehlich (1983: 30) betont einen kognitiv-psychischen Charakter der Sprachverwendung, der sich auf die mentalen Prozesse bezieht und auf der Speicherung und -verarbeitung von Wissen beruht sowie auf dessen Transfer. Aufgrund dieser Bezogenheit auf die Kognition und das Gehirn hat der Sprachgebrauch eine ,intraaktionale Dimension“ (K. Ehlich 1983: 30), gleichzeitig aber geht es hier um die interaktionale Praxis, denn um die oben beschriebenen Tätigkeiten zu realisieren, müssen die Grenzen der Kognition überschritten werden. Erst dann kommt es zu der sprachlichen Interaktion, der verschiedene Ziele zugrunde liegen. Eines der Ziele ist der Wissenstransfer. Eben mit den sprachlichen Mitteln schaffen und konstituieren wir das Wissen; das so geschaffene, vermittelte und erst dann zugängliche Wissen lässt sich dann linguistisch analysieren (S. Grucza 2012: 100). Die Funktion der Fachsprachen beschränkt sich nicht nur darauf, Mittel zur Äußerung von Fachwissen zu sein. Die primäre Funktion von Fachsprachen hat einen kognitiven Charakter und ermöglicht den Menschen, Prozesse der Aneignung, Erzeugung, Strukturierung, Ordnung und Festigung von Fachwissen im menschlichen Gehirn zu realisieren. Die kommunikative Funktion von Fachsprachen, d.h. der Transfer von Fachwissen ist sekundär (S. Grucza 2012: 70, 128). Dank der kognitiven Funktion können die Mitglieder der jeweiligen Fachgemeinschaften neues Fachwissen bilden und altes umorganisieren; kommunikative Funktion erlaubt ihnen das Fachwissen mittels Fachtexte an andere zu transferieren (S. Grucza 2012: 143). Funktional gesehen sind konkrete Fachsprachen also vollständig und autonom. Auch H.-R. Fluck (1996: 239) hat auf die „Vernachlässigung der kognitiven Funktion von Fachsprachen" verwiesen. Es fehlte nach wie vor nach T. Roelcke (2005: 189) an einer richtigen „Theorie- und Methodenbildung auf kommunikationswissenschaftlicher und kognitionslinguistischer Grundlage." Aus dem Grunde soll die Fachsprachenforschung mehr aus der Kognitionspsychologie schöpfen.

Das Fachwissen als Bestandteil der kognitiven Ausstattung eines jeden Fachmanns soll also in den Bereich der Fachsprachenforschung mit einbezogen werden. Die Fachsprachforschung fragt also nicht nur nach fachspezifischen Ausdrucksweisen und Termini, sondern muss nach P. Drewer (2003: 27) „,deren Weltbezug, also das dahinter stehende fachliche Wissen und Modellieren der Welt, mit in die Betrachtungen einbeziehen“. Zu den Aufgaben der Fachsprachenlinguistik gehört nach S. Grucza (2012: 112) die Erforschung der Fachsprachen im engen (instrumentalen) Sinne des Wortes mit den Überlegungen über das Wissen zu integrieren; die Erforschung der kommunikativen Funktionen mit der Erforschung der kognitiven Funktionen der Fachsprachen zu integrieren; die Erforschung über die lexikalische Ebene hinaus auch der weiteren, also der syntaktischen und textuellen Ebenen, auf denen das Fachwissen vertreten ist. Auf keinen Fall solle jedoch das Fachwissen mit sprachlichen Ausdrücken, insbesondere mit Texten gleichgesetzt werden. Die Fachsprachenlinguistik muss ihre Überlegungen und Untersuchungen damit beginnen, was in Wirklichkeit existiert, also mit der Erforschung konkreter Fachleute, die sich 
Fachsprachen bedienen und deren Fachhandlungen zu konkreten Resultaten führen (S. Grucza 2012: 129).

Die Fachleute bzw. Experten und ihr Fachwissen sind Komponenten eines FachBegriffs. Die Fächer sind als institutionalisierte Gebiete bzw. Disziplinen zu verstehen (H. Kalverkämper 1998: 8) und lassen sich nach S. Kühtz (2007: 19) durch „die dort betriebene Auseinandersetzung mit bestimmten, thematisch zusammenhängenden Gegenständen und Fragestellungen“ charakterisieren, wobei Prozesse, Handlungszusammenhänge, Zielsetzungen, Methodologien, Funktionen mitberücksichtigt werden.

Das Fach Medizin, auf das wir uns und im Folgenden konzentrieren, lässt sich laut S. Kühtz (2007: 62) als „ein identifizierbarer, institutionalisierter Tätigkeitsbereich mit eigenen konstituierenden Komponenten" definieren, der durch breit gefächerte und miteinander verflochtene horizontale und vertikale Kommunikationsstrukturen gekennzeichnet ist (vgl. dazu I. Wiese 1994: 14). Eine vollständige horizontale Gliederung der einzelnen medizinischen Fachbereiche lässt sich M.A. Gierzyńska (2015: 136) zufolge jedoch bis heute auf keine fachsprachenlinguistische Auffassung zurückführen. Die Einteilung in theoretische und klinische Fächer entspricht nämlich der medizinischen Praxis. Nach dem Kriterium der Vertikalität werden die Besonderheiten der Fachlichkeit in Bezug auf die kommunikativen Aspekte erfasst. Die klinisch-theoretischen sowie die klinisch-praktischen Fächer sind nicht eindeutig voneinander zu trennen, weil die letzten durch die Benutzung der Fachterminologie mit den ersten stets im unzertrennlichen Verhältnis bleiben, so dass die Theorie- und Praxissprache auch untrennbar miteinander verbunden sind. „Beachtenswert an dieser Stelle ist aber das Auftreten zahlreicher Gemeinsamkeiten und Unterschiede zwischen einzelnen Disziplinen und ihren Fachwortschätzen“ (M.A. Gierzyńska 2015: 136f.).

Auf der Ebene der konstituierenden Elemente unterscheiden sich die Fachsprachen von den sog. Grundsprachen sowie voneinander durch die Fachlexik und die Fachtexttemik (spezifische Textformen und syntaktische Besonderheiten). Was sie miteinander verbindet, ist die Phonemik, Graphemik, Morphemik, Grammatik und nichtfachbezogene Lexik. Fachsprachen sind also keine kompletten, vollständigen Sprachen im linguistischen Sinne des Wortes (S. Grucza 2012: 135). Sie bestehen zum größten Teil aus lexikalischen und syntaktischen Elementen der Gemeinsprache (vgl. dazu M.A. Gierzyńska 2015: 132). Dies trifft auf die Fachsprache der Medizin zu. S. Kühtz (2007: 23) fasst das folgendermaßen zusammen:

Wie alle Fachsprachen ist auch die Fachsprache der Medizin aus der Gemeinsprache hervorgegangen; sie ist und bleibt untrennbar mit der Gemeinsprache verwoben. Die medizinische Fachsprache bildet weder ein eigenständiges Sprachsystem noch eine eigenständige Existenzform von Sprache, sondern sie präsentiert sich als eine spezielle Verwendungsform der Gemeinsprache, die an fachliche Kommunikationssituationen und fachliche Wissensbestände (Fachsemantiken) gebunden ist. Eine partielle Eigenständigkeit weist die Fachsprache der Medizin allenfalls im morphologisch-lexikalischen Bereich [...]. (S. Kühtz 2007: 23) 
M.A. Gierzyńska (2015: 132-3) betont die Tatsache, dass die Gemeinsprache weitgehender Technisierung und Terminologisierung unterliegt, was durch den Einfluss der technisch-wissenschaftlichen Themen auf Lebenspraxis sowie durch den ständigen Kontakt zwischen fachwissenschaftlichen Bereichen verursacht wird. Dies hat dann zur Folge, dass fachbezogene Begriffe und Ausdrucksweisen auf den Laien nicht mehr fremd und unverständlich wirken. T. Ickler (1997: 91) zufolge werden nicht nur die Fachwörter aus der Allgemeinsprache übernommen, sondern wandern auch ihrerseits in die allgemeine Sprache zurück, so dass der Prozess bidirektional verläuft. Eine ähnliche Meinung vertritt W. Seibicke (1959: 76), indem er schreibt: „Fachwörter entstammen ja zum großen Teil der Gemeinsprache, und umgekehrt wandern zahlreiche fachsprachliche Neubildungen in die Gemeinsprache hinüber.“ Das alles zeugt vom dynamischen Charakter der Fachsprachen (vgl. dazu M.A. Gierzyńska 2015: 133). Die Fachsprachenlinguistik bemüht sich eben unter anderem, die Frage danach zu beantworten, wodurch sich die Fachsprache (Fachidiolekt) eines Fachmanns von seinem Alltags-, Gemein-, bzw. Standardsprache (Grundidiolekt) unterscheidet. Eine Antwort auf diese Frage beginnt mit der Analyse entsprechender Fachtexte/Fachäußerungen. Texte sind in dem Maße Fachtexte, in dem sie ein Fachwissen ausdrücken, in welchem Grade sie zu dem Zweck erzeugt werden, um ein Fachwissen darzustellen (S. Grucza 2012: 132f.). Es lässt sich insgesamt sagen, dass die Bestimmung von Fachsprachen nur ausgehend von Fachtexten und mit Bezug auf das Fachwissen möglich ist. Mit Hilfe von Fachtexten erfolgt also der Transfer des Fachwissens. Fachtexte sind sprachliche Objekte, die das Wissen ausdrücken (,,repräsentieren“). Als Fachtexte werden im Folgenden alle konkreten, sprachlichen Äußerungen aufgefasst, die von einem Fachmann in einem konkreten Kommunikationsakt mit der Absicht hervorgebracht werden, einen Ausschnitt seines Fachwissens auszudrücken (S. Grucza 2012: 161ff.). Der Fachtext bezieht sich auf etwas, referiert auf etwas, und zwar auf die Ebene der Denotate (mentale Gegebenheiten) und auf die Ebene der Designate. Jeder Fachtext ist eine konkrete sprachliche Äußerung mit Zeichenfunktion (Ersatzfunktion) für einen Ausschnitt von Fachwissen.

Bei dem Transfer des Fachwissens an die Laien verweist J. Niederhauser (1999: 117ff.; vgl. dazu auch M.A. Gierzyńska 2015: 109) auf für populärwissenschaftliche Präsentation typische Strategien zur Veranschaulichung, zu denen die Personalisierung des fachwissenschaftlichen Wissens, Rückgriff auf die Geschichte der Erforschung des jeweiligen Phänomens, die Betonung des praktischen Nutzens einer Entdeckung, der Gebrauch von Metaphern sowie die „bebilderte“ Sprache, also die Visualisierung von Texten mit Hilfe von Bildern gehören. Text-Bild-Konglomerate ermöglichen eine bessere Vorstellung von komplexen Sachverhalten und abstrakten Begriffen. Texte selbst, all diese Mittel insgesamt erfüllen nämlich die Funktion von Exponenten des Fachwissens, weil ihre Ausdrucksebene ein Ersatz für die denotative Ebene (hier: das Fachwissen) ist (S. Grucza 2012: 153ff.). So bedient man sich unterschiedlicher Verfahren, um die schwer verständlichen fachlichen Sachverhalte einem Laien zugänglich zu machen (vgl. T. Ickler 1997: 322) und um auf diese Weise die Fachkommunikation zu verbessern. 
Die fachwissenschaftliche Kommunikation wird sowohl schriftlich als auch mündlich realisiert, sie wird auch multimedial und multimodal vermittelt. Die Betrachtung der Fachsprachen darf also nicht nur auf den spezifischen Fachstil, bzw. den Fachwortschatz reduziert werden. Es müssen zweifellos weitere Faktoren berücksichtigt werden. Hierzu gehören der Wissensbestand konkreter Personen und ihre Ausbildung sowie die kommunikativen Bedingungen. Dabei schränkt man sich natürlich nicht mehr nur auf den Experten und die ihm zugeschriebenen Merkmale ein. Vielmehr geht man von einer Art Wechselwirkung zwischen der Experten- und Laienwelt aus (vgl. M.A. Gierzyńska 2015: 126).

Unter den Entwicklungstendenzen in der heutigen Fachsprachenforschung verstehen L. Hoffmann und H. Kalverkämper (1998: 363ff.) unter anderem die Auseinandersetzung mit der Fachkommunikation im Hinblick auf die Multimedialität, Interdisziplinarität und Interkulturalität unter besonderer Berücksichtigung der Fachmann-Laie-Kommunikation, die in der letzten Zeit „zu einer der wichtigsten Konstellationen im gesellschaftlichen Informationsaustausch avanciert" ist. Dem Fachmann, im Falle der Fachsprache der Medizin, einem Mediziner, Arzt oder Apotheker, wird als Gegenentwurf ein Laie, in diesem Sinne ein Patient gestellt, dem das Fehlen von Wissen zugeschrieben wird und der daher ,negativ zum Fachmann“ und „zum fachlichen Handeln“ (H. Kalverkämper 1998: 15) definiert wird. Durch die Anerkennung der laienorientierten Kommunikation wird die Laienschaft nicht mehr pejorativ auf der Fachlichkeitsskala eingestuft. Dies gilt für die Sprache der Medizin. Die Berücksichtigung von Laien ist hier einerseits durch das natürliche Miteinander von Experten- und Laienwelt bedingt, andererseits aber resultiert aus dem Status der medizinischen Thematik, die recht häufig in unserem Alltag auftaucht (vgl. M.A. Gierzyńska 2015: 126ff.). S. Kühtz (2007: 29) drückt sich, wie folgt, dazu aus:

Die Kommunikation zwischen dem Arzt und dem Patienten- oder fachsprachenlinguistisch präziser: zwischen Experte und Laie (...) ist „Dreh- und Angelpunkt“ des Faches Medizin und zugleich Ausgangspunkt und Initiator fachlichen Handelns und Expertenseite. Der enge Bezug zur Laienwelt ist konstituierend für das Fach Medizin, wie es sich in einer fachtypischen „Mit-Institutionalisiertheit“ des Laienbezugs zeigt: Sprechstunden, Aufklarungsbroschüren, Patientenvertretungen... aber auch in der Übernahme von Expertenwissen in der Bevölkerung durch zunehmende (massenmediale) medizinische Aufklärung und bei der Aneignung von Handlungswissen (häusliche Blut/Blutzuckermessung, Selbstinjektion von Medikamenten, häusliche Krankenpflege...). (S. Kühtz 2007: 29)

Die Laienebene, die den Nichtfachmann bzw. Nichtkenner mit ihm entsprechendem Wissensbestand vertritt, soll also in der vertikalen Gliederung mitberücksichtigt werden (vgl. S. Wichter 1994: 44). Diese Teilnahme von Laien an der Fachkommunikation hat seinen Grund unter anderem darin, dass die gesundheitliche Problematik in den Alltagsgesprächen präsent ist. Deshalb verfügen auch wenig informierte Laien über ein minimales medizinisches Wissen, das S. Kühtz (2007: 30) ,medizinisches Weltwissen“ nennt. Dazu gehören auch volksmedizinische Kenntnisse und Heilverfahren. In diesem Sinne also wird der Begriff der Laienschaft in die Katego- 
rie der Fachlichkeit integriert, was auch zur Aufhebung der Gegenüberstellung Fachsprache-Gemeinsprache beiträgt. Der Laie, der im Gegensatz zum Experten wenig Merkmale aufweist, wird hier doch S. Kühtz (2007: 30f.) zufolge „zu einem Minimal-Experten" und zugleich zum gleichwertigen, kritischen Kommunikationspartner. Auf diese Weise wird er nicht mehr pejorativ und negativ definiert. Ein gut informierter Laie könnte sogar laut S. Kühtz (2007: 31) in bestimmten Situationen in das Expertenfeld vorrücken, so dass „Experten und Laienstatus sich sogar umkehren können.“ Nach M.A. Gierzyńska (2015: 143) unterscheiden sich Laie und Experte, hier Patient und Arzt, voneinander: in der Sprachverwendung sowie in der Beschaffenheit der Wissensbestände. Die beiden Welten existieren nebeneinander, bezogen auf das Fach Medizin und auf die Sprache der Medizin (vgl. M.A. Gierzyńska 2015: 143).

Mit Hilfe der Sprache der Medizin erfüllt man verschiedene kommunikative Aufgaben von Forschung über Lehre bis Gesundheitsaufklärung auf allen Abstraktionsebenen (vgl. I. Wiese 1998: 710). Um medizinische Texte unterscheiden zu können, benötigen wir ,die Merkmale Struktur, Funktion und Kommunikationssituation“ (S. Kühtz 2007: 58). In Anlehnung an M.A. Gierzyńska (2015: 150 ff.) können wir im Expertenkreis u.a. die in den Fachzeitschriften publizierten Originalarbeiten, Kaustiken (Fallberichte), Übersichtsarbeiten, kommentierte Referate unterscheiden. Für die Aus- und Weiterbildung dienen Nachschlagewerke, wie Hand- und Lehrbücher, Leitfäden, Atlasse, medizinische Wörterbücher oder Lexika. Innerhalb der medizinischen Praxis finden wir Krankengeschichten, Arztbriefe, Krankenscheine, Operationsberichte, Totenscheine sowie Arzneimittelrezepte, die als formal und sprachlich hoch standardisierte Informations- und Dokumentationstexte verstanden werden. $\mathrm{Zu}$ den laienorientierten Textsorten gehören Aufklärungsbogen bzw. Merkblätter, Broschüren, Medikamentenpackungsbeilagen, Ratgeber sowie medizinische Aufklärungstexte in Zeitungen, Illustrierten und Gesundheitsmagazinen, auch online (vgl. I. Wiese 1998: 1278; 2000: 710ff.). Dabei geht es laut J. Niederhauser (1997: 109) nicht nur um die Vermittlung der Erkenntnisse an die Laienschaft, sondern auch „um einen spezifischen Typ des Textproduzierens“, nämlich um das Umschreiben wissenschaftlicher und fachlicher Darstellungen für Interessengruppen außerhalb des Faches und der Wissenschaft. Für die Unterscheidung von Fachtextsorten sollte die Definition von T. Roelcke (2005: 42) ausschlaggebend sein, in der sie als Typen oder Klassen von Fachtexten aufgefasst werden, die ,im Rahmen bestimmter Verwendungsweisen innerhalb der fachlichen Kommunikation jeweils bestimmte funktionale und formale Gemeinsamkeiten aufweisen“".

Einen Klassifikationsversuch der medizinischen Texte unternimmt P. Löning (1981: 82ff.). Dieses Modell präsentiert verschiedene Fachlichkeitsgrade von Texten in Hinblick auf die Kommunikationspartner. Es wird zugleich eine vertikale Schichtung der Fachsprache der Medizin dargestellt, indem medizinische Texte klassifiziert werden. Die Autorin unterscheidet die vier Ebenen: schriftlich-mündlich in Wechselwirkung zu theoretisch-praktisch. Sie lassen sich dann in mehrere Stile aufteilen, wenn die Kriterien des Fachlichkeitsgrades der an der Kommunikation beteiligten Personen und das Ziel des Textes bzw. Gesprächs berücksichtigt werden, wo- 
rauf näher bei der Textsortenklassifizierung eingegangen wird. Auf allen vier Ebenen ist die Kommunikation 1) unter den Fachwissenschaftlern, 2) zwischen Fachleuten und den Halbfachleuten 3) sowie zwischen Fachleuten und den Laien 4) und schließlich unter den Laien möglich. Es ergibt sich daraus z.B. auf der theoretischschriftlichen Ebene der Stil der wissenschaftlichen Texte, der Belehrung, der Aufklärung und der Stil der populärwissenschaftlichen Texte; auf der theoretischpraktischen Ebene der Stil der Information, der Anweisung und der Anordnung in den Vordergrund (vgl. dazu M.A. Gierzyńska 2015: 151). Wie P. Löning (1981: 84) selbst bemerkt, scheinen unter anderem die Stile der Belehrung und Aufklärung die interessantesten Fälle zu sein, da sie den Aspekt der Vermittlung und Erklärung des Wissens an Nichtfachleute thematisieren. Die mündliche Ebene entspricht in jeder Hinsicht den Kommunikationspartnern und den Zielen der schriftlichen Ebene. Der Unterschied besteht nur in der Art der Darstellung, die diese Stile prägt. Eine wissenschaftliche Diskussion und ein belehrender Vortrag sowie ein einfaches Gespräch könnten hier als relevante Beispiele dienen.

Die fachsprachliche Kommunikation zwischen Experten und Laien verläuft heutzutage immer häufiger internetbasiert durch die sog. Neuen Medien, und zwar nicht nur sprachlich, sondern auch visuell. Der konkrete Text wird sehr oft bebildert/visualisiert, so dass eine funktionale Einheit zusammen mit dem Bild entsteht. Darüber hinaus gibt es auch kurze Filme, die verschiedene biologische Prozesse präsentieren sowie Diagramme, grafische Darstellungen und Modelle. Viele gesundheitliche online-Magazine, wie z.B. www.onmeda.de, www.gesundheit.de, www.medizin.de, sind darauf eingestellt, folgende Funktionen zu erfüllen: das Fachwissen an die Laien zu vermitteln, sie aufzuklären, zu belehren sowie praktisch anzuleiten. Hier übernimmt auch das Bild eine wichtige Funktion und steht mit dem Text in einer gut konzipierten, wechselartigen Verflechtung. Den beiden Elementen kommen verschiedene Aufgaben zu und sie tragen auf eine andere Art und Weise zum gemeinsamen Sinn bei. Wo Bilder und Texte am selben Ort platziert werden, sucht der Betrachter einen sinnvollen Zusammenhang. Die Grundlage für die Integration von beiden Elementen ist ihre Verteilung auf der Fläche, also das räumliche Nebeneinander, ihre gegenseitigen Größenanteile und räumlichen Konfigurationen. Die Integration dieser Elemente geht jedoch über die visuell-räumliche Ebene hinaus und realisiert sich vollkommen auf der begrifflichen Ebene, denn beide - das Bild und das Wort - aktivieren im Gehirn Begriffe bzw. Assoziationen. Indem das Fachwissen auf den Internetportalen für Medizin und Gesundheit transferiert wird, kommt oft dazu, dass die Inhalte nicht nur visualisiert, sondern auch trivialisiert werden. Es sei an dieser Stelle ein Beispiel angeführt: 


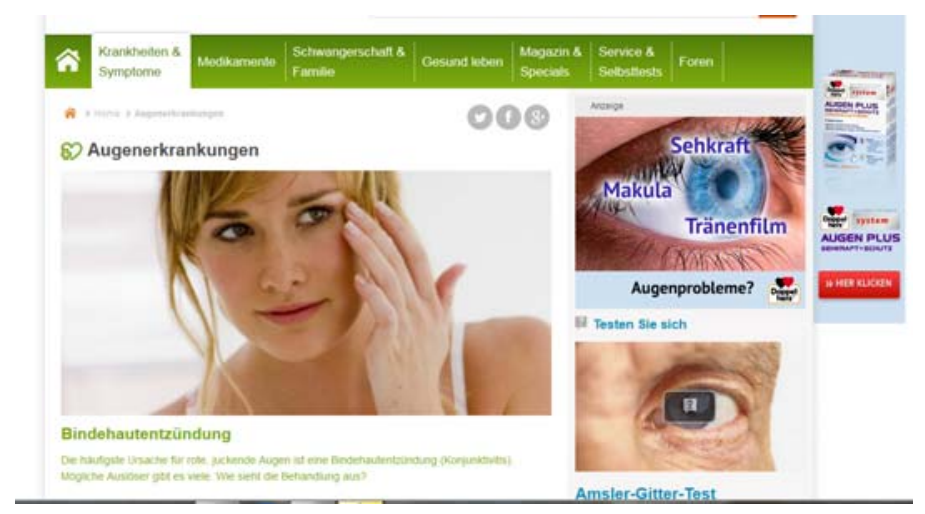

Abbildung 1: www.onmeda.de (abgerufen am 20.06.2015 um 19.27)

Krankheiten und Symptome werden alphabetisch zusammengestellt. Als die erste unter den Augenkrankheiten erscheint Bindehautentzündung. Auf dieser konkreten Webseite überwiegen visuelle Elemente, und zwar rechts oben finden wir die Werbung für Augentropfen, rechts unten kann man einen Amsler-Gitter-Test durchführen und schließlich auf der linken Seite geht es um die zu beschreibende Krankheit. Der Text unter dem Bild besteht nur aus drei Sätzen, wobei man anmerken muss, dass das Bild in diesem Fall überhaupt nicht informativ ist und sogar als überflüssig empfunden werden kann. Das Bild ergänzt den Text, ohne ihm jedoch etwas entscheidend Neues hinzuzufügen. Ohne den Text könnte man es überhaupt nicht identifizieren: der Text konkretisiert also dieses Bild, mit anderen Worten: monosemiert. Die weiteren, fotografischen Bilder dienen nicht mehr bloß der Illustration, sondern ergänzen die jeweiligen textuellen Informationen, wie in dem Beispiel unten, wo das Bild mit dem kurzen Text korrespondiert und eigentlich als Kon-Bild zu dem Text fungiert. Die Wirkung dieses Bildes ist sehr stark, seine Informativität und seine Überzeugungskraft sehr hoch. Er dient sogar als Beweis und sein Potenzial besteht in einer visuellen Argumentation:

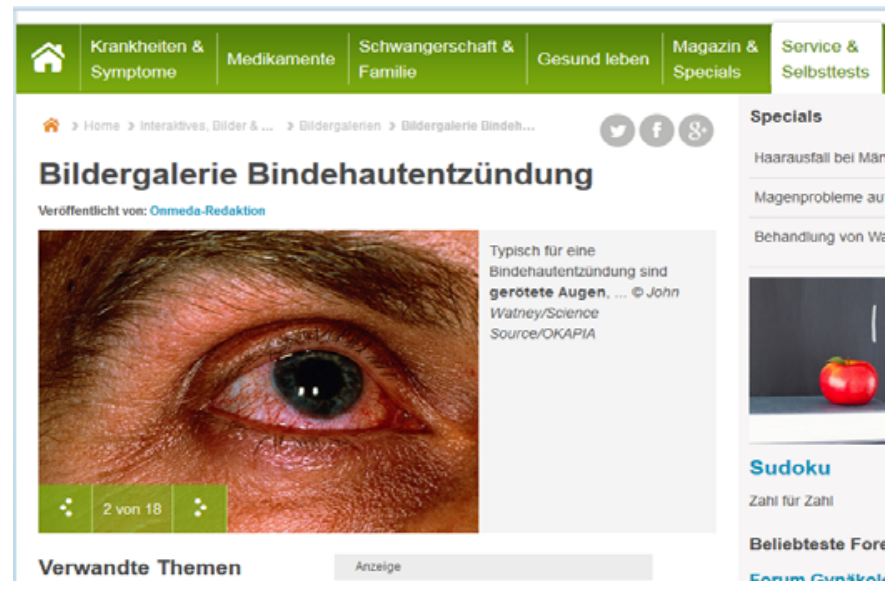

Abbildung 2: www.onmede.de (abgerufen am 20.06.2015 um 19.27) 
Mit Texten und Bildern werden Bedeutungen auf unterschiedliche Weise vermittelt; indem sie sich aufeinander beziehen, bilden sie einen Komplex, aus dem dann eine neue Bedeutungsganzheit erschlossen werden kann. Durch die Zusammenstellung mit dem Bild gewinnen Texte Evidenz (M. Steinseifer 2010: 342ff.), die er auch als Prägnanz bezeichnet. Ein gegenständliches Bild verleiht dem, was es im durch Textteile mitkonstituierten Zusammenhang darstellt, zusätzliche Plausibilität. Dies realisiert sich vollkommen in dem obigen Beispiel. M. Steinseifer (2010: 328) spricht auch von einer Dopplung (Redundanz), wenn das Bild einen Teil der Bedeutung verdoppelt, die mit sprachlichen Mitteln ausgedrückt wird. Warum also werden sie in solchen Fällen verwendet? Der Grund liege in der Pragmatik, und zwar in der Überzeugungskraft von Bildern.

Bilder verschiedener Art beziehen sich auf die textuellen Elemente, wobei sich dieses Verhältnis als reziprok bezeichnen lässt. Der gesamte Sinn geht nach U. Schmitz (2003: 256f.) aus ihrem Zusammenschluss hervor. Bilder können die Situation ersetzen, die in der Kommunikation schon vorhanden ist und die sprachlich nicht mehr artikuliert zu werden braucht. Sie werfen eine Bedeutung auf Texte, die bei ihnen stehen, ähnlich wie Worte in mündlicher Kommunikation von der umgebenden Situation her mitverstanden werden. In dem online-Transfer des Fachwissens haben wir es am häufigsten mit der Kongruenz von Text und Bild zu tun, wenn sie denselben Begriff hervorrufen und wenn sie gleichwertige, einander ergänzende Elemente sind. Text und Bild stehen oft in einem Subsidiärverhältnis, wenn das Bild als Illustration den Text unterstützt, oder umgekehrt der Text als Kommentar das Bild. Inhaltlich sind sie komplementär, denn beide sind gleichwertige Elemente und als solche basieren auf einem gemeinsamen Wissensschema im Langzeitgedächtnis. Durch dieses Schema verknüpfen sie sich schließlich, so dass das Bild die Textverarbeitung unterstützt oder der Text die Bildverarbeitung. Auf diese Weise ergänzen sich Text und Bild und aus ihrem Nebeneinander entsteht eine holistische Gesamtbotschaft (E. Żebrowska 2013: 204 ff., S.P. Ballstaedt 2005: 66, S.F. Sager 2000: 592).

Sehr oft konkretisiert auch das Bild den Text und ergänzt ihn um Details, wie in dem folgenden Beispiel:

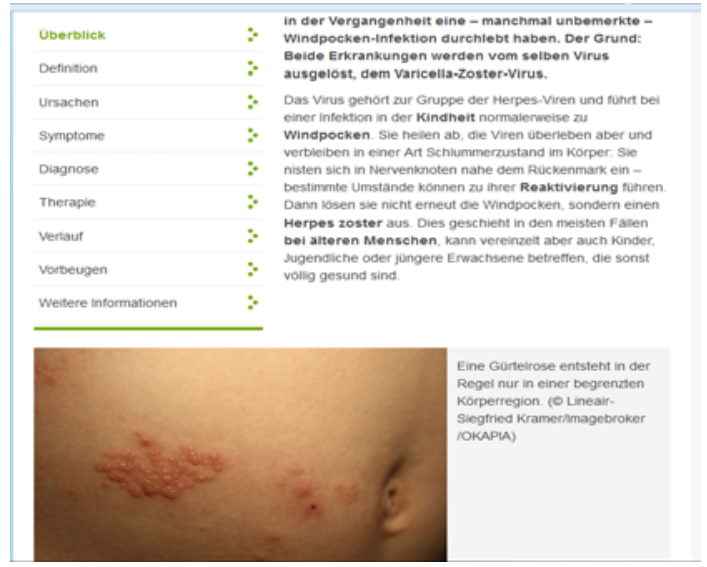

Abbildung 3 
Die Rede ist von einer Gürtelrose, die zu den zehn häufigsten Krankheiten eingestuft worden ist. Es wird ein Überblick über die Krankheit in Form einer längeren Beschreibung angeboten, die Definition, Ursachen, Symptome, Diagnose, Therapie beinhaltet. Auf der dargestellten Webseite macht das Bild den Text eindeutig und beweist ihn zugleich. Die Krankheit wird durch weitere Bilder illustriert, darunter auch durch Modelle der Viren:

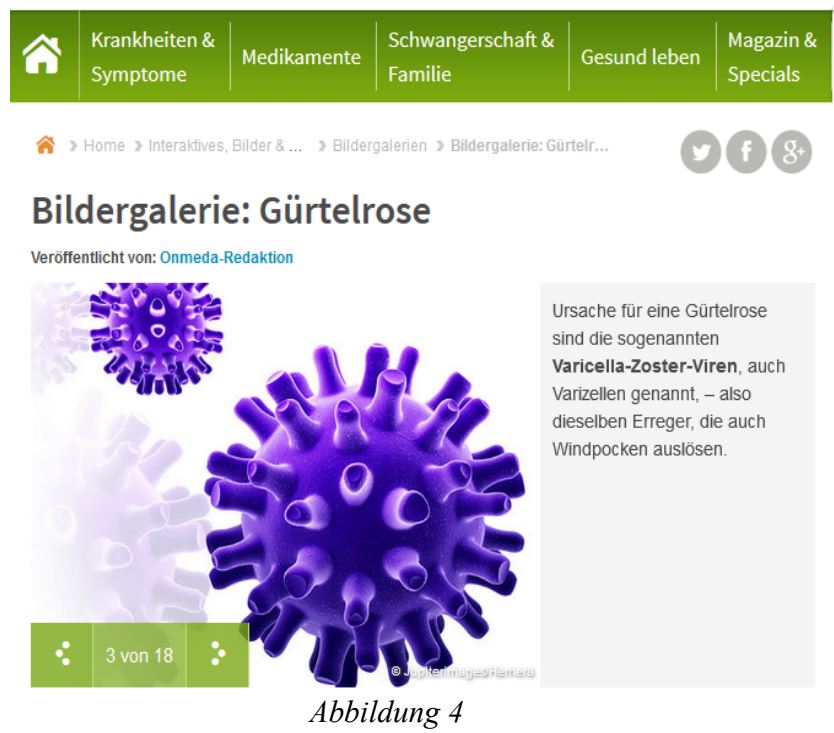

Insgesamt lässt sich sagen, dass moderne Kultur immer mehr zu einer visuellen Kultur tendiert. Bei dem Transfer von Fachwissen in den internetbasierten Hypertexten, zu denen gesundheitliche online-Magazine ohne Weiteres zählen, spielen sowohl Texte als auch Bilder eine wichtige Rolle. Es kann sein, dass beide ungefähr dasselbe vermitteln, so dass die Information verdoppelt wird. Sie können auch wechselseitig und gemeinsam zum Gesamtverständnis beitragen oder sich gegenseitig ergänzen, wobei eins der Elemente dominierend ist. Möglich ist auch, dass sie sich inhaltlich sehr unterschieden, aber doch integrativ verwendet werden. Mit einem Wort also: es werden bestimmte Methoden der Visualisierung von sprachlichen Aussagen gebraucht, vor allem: Ähnlichkeit (visuelle Analogie), Beweis (visuelle Argumentation), Gedanken-Verknüpfung (visuelle Assoziation), Teil-für-Ganzes (visuelle Synekdoche), Grund-Folge (visuelle Kausal-/Instrumental-Relation), Wiederholung (visuelle Repetition), Steigerung (visuelle Gradation), Hinzufügung (visuelle Addition).

\section{Bibliographie}

Antos, G. (2003), Wie kann sich die Linguistik Öffentlichkeit ıschaffen«? In: A. Linke/ H. Ortner/ P. Portmann-Tselikas (Hrsg.), Sprache und mehr. Ansichten einer Linguistik der sprachlichen Praxis. Tübingen. 
Antos, G. (2007), „, Texte machen Wissen sichtbar!“ Zum Primat der Medialität im Spannungsfeld von Textwelten und (inter-)kulturellen Wirklichkeitskonstruktionen. In: F. Grucza/ M. Olpińska/ H.-J. Schwenk (Hrsg.), Germanistische Wahrnehmungen der Multimedialität, Multilingualität und Multikulturalität. Warszawa, 34-45.

Ballstaedt, S.P. (2005), Text-Bild-Kompositionen im Unterrichtsmaterial. In: Der Deutschunterricht 4/2005, 61-70.

Drewer, P. (2003), Die kognitive Metapher als Werkzeug des Denkens. Zur Rolle der Analogie bei der Gewinnung und Vermittlung wissenschaftlicher Erkenntnisse. Tübingen.

Ehlich, K. (1983), Text und sprachliches Handeln. Die Entstehung von Texten aus dem Bedürfnis nach Überlieferung. In: A. Assmann/ J. Assmann/ C. Hardmeier (Hrsg.), Schrift und Gedächtnis. Beiträge zur Archäologie der literarischen Kommunikation. München, 24-43.

Fluck, H. R. (1996), Fachsprachen: Einführung und Bibliographie. Tübingen.

Gierzyńska, M.A. (2015), Konzeptuelle Metaphern in medizinischen Texten. Olsztyn.

Grucza, S. (2012), Fachsprachenlinguistik. (Warschauer Studien zur Germanistik und zur Angewandten Linguistik; 1), Frankfurt a. M. ect.

Hoffmann, L./ H. Kalverkämper/ H.E. Wiegand (1998) (Hrsg.), Fachsprachen. Ein internationales Handbuch zur Fachsprachenforschung und Terminologiewissenschaft. Berlin/New York.

Ickler, T. (1997), Die Disziplinierung der Sprache. Fachsprachen in unserer Zeit. Tübingen.

Kalverkämper, H. (1998), Fach und Fachwissen. In: Hoffmann et al. (Hrsg.), 1-24.

Kalverkamper, H. (1998), Fachsprache und Fachsprachenforschung. In: L. Hoffmann et al. (Hrsg.), 48-60.

Kühtz, S. (2007), Phraseologie und Formulierungsmuster in medizinischen Texten. Tübingen.

Löning, P. (1981), Zur medizinischen Fachsprache. Stilistische Gliederung und Textanalysen. In: Muttersprache 91, 79-92.

Niederhauser, J. (1999), Wissenschaftssprache und populärwissenschaftliche Vermittlung. (Forum für Fachsprachen- Forschung; 53), Tübingen.

Niederhauser, J. (1997), Das Schreiben populärwissenschaftlicher Texte als Transfer wissenschaftlicher Texte. In: E. Jakobs/ D. Knorr (Hrsg.), Schreiben in den Wissenschaften. Frankfurt a. M. etc., 107-124.

Roelcke, T. (2005), Fachsprachen. Berlin.

Schmitz, U. (2003), Text-Bild-Metamorphosen in Medien um 2000. In: U. Schmitz/ H. Wenzel (Hrsg.), Wissen und neue Medien. Bilder und Zeichen von 800 bis 2000. Berlin, 241-263.

Sager, S.F. (2000a), Hypertext und Hypermedia. In: K. Brinker/ G. Antos/ W. Heinemann/ S.F. Sager (Hrsg.), Text- und Gesprächslinguistik. Linguistics of Text and Conversation. Ein internationales Handbuch zeitgenössischer For- 
schung. An International Handbook of Contemporary Research. 1. Halbband. Berlin/New York, 587-603.

Seibicke, W. (1959), Fachsprache und Gemeinsprache. In: Muttersprache 69, 7084.

Steinseifer, M. (2010), Prägnanzen. Bilder und ihre Effekte in der pragmatischen Linguistik. In: A. Deppermann/ A. Linke (Hrsg.), Sprache intermedial. Stimme und Schrift, Bild und Ton. Berlin/New York, 325-358.

Wichter, S. (1994), Experten- und Laienwortschatz. Versuch einer Lexikologie der Vertikalität. (Reihe Germanistische Linguistik; 144). Tübingen.

Wiese, I. (1994), Medizinische Fachsprache und Fachlexikographie - Theoretische Aspekte. In: S. Dressler/ B. Schaeder (Hrsg.), Wortebücher der Medizin. Beitrage zur Fachlexikographie, Tübingen.

Wiese, I. (1998): Die neure Fachsprache der Medizin seit der Mitte des 19. Jahrhunderts unter besonderer Berücksichtigung der Inneren Medizin. In: L. Hoffmann et al. (Hrsg.), 1278-1285.

Wiese, I. (2000), Textsorten des Bereichs Medizin und Gesundheit. In: K. Brinker/ G. Antos/ W. Heinemann/ S.F. Sager (Hrsg.), Text- und Gesprächslinguistik. Ein internationales Handbuch zeitgenössischer Forschung, Berlin/New York, 710718.

Żebrowska, E. (2013), Text - Bild - Hypertext. (Warschauer Studien zur Germanistik und zur Angewandten Linguistik; 10), Frankfurt a. M. etc. 